\title{
Rapid identification and quantitation of the viable cells of Lactobacillus casei in fermented dairy products using an aptamer-based strategy powered by a novel cell-SELEX protocol
}

\author{
Shixi Song, ${ }^{1}$ Xingyu Wang, ${ }^{1 *}$ ๑ $\mathrm{Ke} \mathrm{Xu},{ }^{2}$ Lufang Ning, ${ }^{1}$ and Xingbin Yang ${ }^{1 *}$ \\ ${ }^{1}$ Shaanxi Engineering Laboratory for Food Green Processing and Safety Control, Shaanxi Key Laboratory for Hazard Factors Assessment \\ in Processing and Storage of Agricultural Products, College of Food Engineering and Nutritional Science, Shaanxi Normal University, \\ Xi'an 710062, Shaanxi, China \\ ${ }^{2}$ Department of Joint Surgery, Hong Hui Hospital, Xi'an Jiaotong University, Xi'an 710054, Shaanxi, China
}

\section{ABSTRACT}

An aptamer-based strategy was developed for qualitative and quantitative analysis of viable Lactobacillus casei in dairy products. Three highly specific aptamers for $L$. casei were obtained using systematic evolution of ligands by exponential enrichment protocol using the whole bacterium cell as the target (cell-SELEX) facilitated by polyethyleneglycol and chitosan modified graphene oxide and complementary ring-mediated rolling circle amplification. Two aptamers, one for separating and enriching the L. casei cells and the other for generating fluorescence signals, were employed to develop an aptamer-based strategy, which was demonstrated for the selective detection of $L$. casei in commercial dairy drinks, with a dynamic range of $10^{5}$ to $10^{9} \mathrm{cfu} / \mathrm{mL}$. Viable and nonviable $L$. casei cells could be discriminated based on the significant difference in fluorescence intensity. This established strategy is of high selectivity and sensitivity, and can be used for rapid analysis of viable L. casei in quality control and food surveillance areas. Key words: Lactobacillus casei, aptamer, graphene oxide, viable cell

\section{INTRODUCTION}

Lactic acid bacteria (LAB) are considered probiotics, which were defined by FAO/WHO (2002) as "live microorganisms able to confer a health benefit on the host when administered in adequate amount." Lactic acid bacteria are frequently used in producing

Received March 27, 2019.

Accepted August 8, 2019.

*Corresponding authors: wangxingyu@snnu.edu.cn and xbyang@ snnu.edu.cn fermented dairy products such as yogurt, kefir, cheese and acidophilus milk (Reid and Bocking, 2003; Kourkoutas et al., 2005; Jankovic et al., 2010). Among LAB, Lactobacillus casei has been extensively added to the milk-derived food for its superior probiotic properties including immunomodulation, anti-infection effects, reduction of serum cholesterol, alleviation of lactose maldigestion, and so on (Yadav et al., 2007). To ensure the functional effects exhibited by $L$. casei, the amount of the bacterium in food products is a matter of concern. It is generally accepted that viable $L$. case $i$ has to reach $10^{6} \mathrm{cfu} / \mathrm{mL}(\mathrm{g})$ as a minimal level and $10^{7}$ to $10^{8} \mathrm{cfu} /$ $\mathrm{mL}(\mathrm{g})$ as a satisfactory level to survive the acidic conditions of the upper gastrointestinal tract and proliferate in the intestine (Boylston et al., 2004; Sohrabvandi et al., 2010; Bagheripoor-Fallah et al., 2015). Many dairy drinks containing $L$. casei are available on the market. However, substitution of $L$. case $i$ with other probiotic LAB such as Lactobacillus acidophilus and Lactobacillus bulgaricus for commercial profit may be happening, which is regarded as consumer fraud and legislatively banned worldwide. Therefore, rapid enumeration and identification of $L$. casei from other closely related microorganisms in dairy food products is of great significance for quality assurance and market supervision.

Traditionally, L. casei in food products was analyzed by the plate-counting cultured on the selective medium (Temmerman et al., 2004). Although the method was considered classic, the long process and complicated operation failed to meet the requirement of rapid detection. To enhance efficiency of the analysis, many molecular approaches have been developed based on the genetic information of L. casei. The PCR-based methods, such as pulsed-field gel electrophoresis, amplified fragment length polymorphism, amplified ribosomal DNA restriction analysis, and multilocus sequence typing can identify the bacterium in a relative short period. However, false-positive results caused by cross-contamination often occur, and complexity 
of extracting DNA from L. casei is still a limitation for rapid detection (Furet et al., 2004). On the other hand, several non-PCR-based approaches (genetic probes, ribotyping, macrorestriction enzyme analysis) were developed, and were demonstrated to be accurate, time efficient, and capable of identifying a defined LAB in a single reaction (Singh et al., 2009). Nonetheless, their reliance on expensive apparatus and well-trained technicians hamper their widespread application. Most importantly, those DNA-based strategies were not able to distinguish viable and nonviable $L$. casei because the dead and dormant cells still possess the integrated genomic DNA, a fact that probably leads to false enumeration results (Davis, 2014).

An aptamer is a single-stranded DNA (ssDNA) or RNA oligonucleotide that is able to specifically bind to various targets such as metal ions, toxins, proteins, and other biological macromolecules (Duan et al., 2016). Basically, an aptamer is selected from a huge stochastic library containing $10^{12}$ to $10^{17}$ ssDNA or RNA sequences by repetitive binding of the oligonucleotides to the target, a process called systematic evolution of ligands by exponential enrichment (SELEX; Lee et al., 2012). Recently, Sefah et al. (2010) reported an advanced SELEX protocol using the whole bacterium cell as the target (cell-SELEX), and the strategy has been used in cancer diagnosis and bacteria detection. The bacteria-specific aptamers are able to bind to transmembrane proteins on the cell surface, with the affinity comparable to that of antibodies (Shi et al., 2011).

In this study, we developed an aptamer-based strategy for qualitative and quantitative analysis of viable $L$. casei in dairy-based drinks. First, the L. casei-specific aptamers were obtained by using a novel cell-SELEX method that uses polyethyleneglycol (PEG) and chitosan functionalized graphene oxide (PC-GO) to eliminate nonspecific ssDNA sequences, and an isothermal amplification technology (complementary ring-mediated rolling circle amplification, CRM-RCA) instead of PCR to amplify the potential aptamer sequences. The selected aptamers were proved to be of high affinity specifically to $L$. casei other than other bacteria strains of LAB. Second, 2 aptamers, one for specifically enriching $L$. casei from the food matrix (enriching aptamer, E-Apt) and the other for detecting the living cells (detecting aptamer, D-Apt), were employed here to establish a detection strategy with the ability to distinguish viable and nonviable $L$. casei in a linear range of $10^{5}$ to $10^{9} \mathrm{cfu} / \mathrm{mL}$. The method developed here was demonstrated to be accurate and efficient, which enables it to be used for rapid identifying and enumerating of $L$. casei in dairy products.

\section{MATERIALS AND METHODS}

\section{Reagents}

de Man, Rogosa, and Sharpe (MRS) agar and broth media were purchased from Aobox Biotechnology Co., Ltd. (Beijing, China). The BBL and M17 broth and agar media were provided by Hopebio Biotechnology Co., Ltd. (Qingdao, China). All of the oligonucleotides used in the study were synthesized and purified with HPLC by Sangon Company (Shanghai, China). The T4 DNA ligase, Phi29 DNA polymerase, dNTP, and 50-bp DNA ladder were provided by Thermo Scientific (Pittsburgh, PA). The PBS solution was obtained from HyClone (South Logan, UT). Streptavidin magnetic beads (SMB), Nb.BbvCI, and Nt.BbvCI were purchased from New England Biolabs (Ipswich, MA). Bovine serum albumin and Tween-20 were provided by Solarbio Science \& Technology Co. Ltd. (Beijing, China). Lactobacillus casei (ATCC 393), L. acidophilus (ATCC 4356), Streptococcus thermophilus (ATCC 19258), L. bulgaricus (ATCC 11842), Bifidobacterium lactis (ATCC SD5219), and Lactobacillus plantarum (ATCC 8014) were provided by the American Type Culture Collection (ATCC, Manassas, VA). The solutions used in this study were prepared by Milli-Q purified water (resistance $<18.2 \mathrm{M} \Omega \cdot \mathrm{cm}^{-1}$ ). The synthetic route of PC-GO composites used in cell-SELEX to eliminate ssDNA with lower affinity to $L$. casei can be found in the Supplemental Information (https://doi .org/10.3168/jds.2019-16693).

\section{Bacteria Culture}

The MRS broth was used to culture L. casei and L. plantarum. The MRS broth with the addition of maltose was prepared for L. acidophilus. Streptococcus thermophilus and L. bulgaricus were cultured under anaerobic conditions using M17 and MRS media, respectively. The B. lactis was cultured by using BBL medium. All the liquid cultures were incubated at $37^{\circ} \mathrm{C}$ overnight with a shaking speed of $120 \mathrm{rpm}$. The growth curve of $L$. casei can be seen in Supplemental Figure S1 (Supplemental Information; https://doi.org/ 10.3168/jds.2019-16693). For the colony-forming unit test, each bacterium was cultured in the corresponding broth for $18 \mathrm{~h}$. The result of colony-forming unit counts of different dilution can be seen in Supplemental Table S1 (https://doi.org/10.3168/jds.2019-16693). The cell suspension was diluted by a gradient from $10^{-1}$ to $10^{-9}$. Then $20 \mu \mathrm{L}$ of each gradient was plated on the corresponding agar dish, followed by incubation at 
$37^{\circ} \mathrm{C}$ for $24 \mathrm{~h}$ to allow the bacterium to fully grow. The colonies were then counted. Each dilution gradient was performed in triplicate and the average number was recorded.

\section{Aptamer Selection}

Aptamer selection followed a similar strategy as previously described (Song et al., 2019a). Lactobacillus casei was used as the target for cell-SELEX. Certain amount of cells were suspended in $1 \mathrm{~mL}$ of PBS to guarantee a final concentration of $10^{6} \mathrm{cfu} / \mathrm{mL}$. Cells were centrifuged at $16,000 \times g$ at $4^{\circ} \mathrm{C}$ for 6 min to remove the PBS and washed twice with $1 \times$ binding buffer (BB) consisting of $137 \mathrm{mM} \mathrm{NaCl}, 2.7 \mathrm{mM} \mathrm{KCl}$,

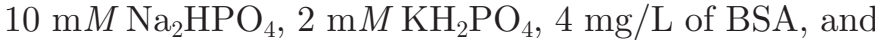
$0.05 \%$ (vol/vol) Tween-20. The SELEX was initiated with the randomized ssDNA library (250 pmol), and amplified aptamer pool (50 pmol) was used in the subsequent rounds. A total of $10^{6} \mathrm{~L}$. casei cells were used for each SELEX cycle. The same number of cells of S. thermophilus, L. bulgaricus, and L. plantarum were used as the counter targets.

The washed cells were recollected and resuspended by $400 \mu \mathrm{L}$ of $1 \times \mathrm{BB}$ containing a certain amount of ssDNA. The suspension was incubated at $37^{\circ} \mathrm{C}$ for 30 min, enabling the potential aptamers to bind with the target cells. To eliminate the nonspecific ssDNA bound to the tube wall, the suspension was transferred to a new Eppendorf tube after the incubation. Then $2.0 \mathrm{mg}$ of PC-GO was introduced to the suspension, and the resulting mixture was incubated at $37^{\circ} \mathrm{C}$ for $10 \mathrm{~min}$. Then the mixture was centrifuged at $7,000 \times g$ at $4^{\circ} \mathrm{C}$ for $5 \mathrm{~min}$, the supernatant containing PC-GO loading ssDNA with lower affinity to $L$. casei was discarded, and the cell precipitate was washed twice using $1 \times$ $\mathrm{BB}$. Then the cells were resuspended with $1 \times$ dissociating buffer containing $137 \mathrm{mM} \mathrm{NaCl}, 2.7 \mathrm{mM} \mathrm{KCl}$, $10 \mathrm{~m} M \mathrm{Na}_{2} \mathrm{HPO}_{4}, 2 \mathrm{mM} \mathrm{KH_{2 }} \mathrm{PO}_{4}$, and $0.05 \%$ (vol/vol) Tween-20, followed by heating at $95^{\circ} \mathrm{C}$ for $5 \mathrm{~min}$ to denature and dissociate the cell-binding aptamer candidates (CAC). The cell suspension was immediately centrifuged at $16,000 \times$ gat $4^{\circ} \mathrm{C}$ for $5 \mathrm{~min}$, and the supernatant containing dissociated $\mathrm{CAC}$ was collected. The CAC were amplified by CRM-RCA as illustrated in Supplemental Scheme S1 (https://doi.org/10.3168/ jds.2019-16693), and the detailed operation process can be found in the Supplemental Information (https: //doi.org/10.3168/jds.2019-16693). The amplification products in single-stranded form were directly used as aptamer pool in the next round of selection. Six rounds of selection interposed with 2 counter-screening cycles were performed, and the CAC from the last round of selection were cloned and sequenced to obtain the aptamer sequences.

\section{Flow Cytometry Analyzing Specificity of the Selected Aptamers}

Flow cytometry analysis followed a similar strategy as previously described (Song et al., 2019a). A BD FACSCalibur flow cytometer (BD Biosciences, San Jose, CA) was used to evaluate the specificities of the selected aptamers to 6 different LAB strains (L. casei, L. acidophilus, S. thermophilus, L. bulgaricus, B. lactis, and $L$. plantarum). The $5^{\prime}$ fluorescein isothiocyanate (FITC)-labeled aptamers were denatured at $95^{\circ} \mathrm{C}$ for $5 \mathrm{~min}$ to denature the secondary structure formed by self-folding. The FITC-labeled aptamers of $100 \mathrm{n} M$ were incubated with bacterial cells of $10^{6}$ at $37^{\circ} \mathrm{C}$ for $30 \mathrm{~min}$ in a total volume of $500 \mu \mathrm{L}$ of $1 \times \mathrm{BB}$. The cells loading fluorescent aptamers were washed once by $1 \times \mathrm{BB}$, followed by resuspension and immediate flow cytometric analysis. Forward scatter, side scatter, and fluorescence intensity were measured, and the results of cells incubated with fluorescently labeled ssDNA library were used as control. For the dissociating constant $\left(\boldsymbol{K}_{\boldsymbol{d}}\right)$ assay, aptamers varying in concentration $(0-150 \mathrm{n} M)$ were incubated with a fixed number of $L$. casei cells $\left(10^{6}\right)$. Flowing software 2.5.1 (Perttu Terho, Finland) was used to interpret and present the data.

\section{Secondary Structural Analysis of the Selected Aptamers}

The secondary structure of each aptamer sequence was predicted using mfold online application (http:/ /unafold.rna.albany.edu/?q=mfold) with input condi-

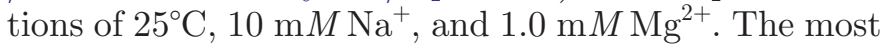
likely structure was chosen on the basis of the lowest predicted free energy of formation $(\Delta G, \mathrm{kcal} / \mathrm{mol})$.

\section{Establishing the Aptamer-Based Strategy to Specifically Detect L. casei}

To quantitatively detect the presence of $L$. casei, 2 aptamers, E-Apt and D-Apt, binding to different regions of the cell surface, were used. The E-Apt was obtained by adding a poly(A) tail with biotin moiety at $3^{\prime}$ terminus of Apt-2, whereas a fluorescent FITC group was attached to the $5^{\prime}$ end of Apt-3 to construct D-Apt. Bacteria suspensions varying in amount were mixed with E-Apt of $0.05 \mu M$ in a final volume of 100 $\mu \mathrm{L}$ of $1 \times \mathrm{BB}$ and incubated at $37^{\circ} \mathrm{C}$ for $30 \mathrm{~min}$. Then SMB of $10 \mu \mathrm{L}$ was added, and the solution was kept at 
room temperature for another 5 min with intermittent shaking. The SMB loading bacteria via E-Apt were then collected by magnet separation. Then $40 \mu \mathrm{L}$ of D-Apt $(0.05 \mu M)$ in $1 \times \mathrm{BB}$ was used to resuspend the collected cells, and the suspension was incubated at $37^{\circ} \mathrm{C}$ for $30 \mathrm{~min}$, followed by washing twice with $1 \times$ BB. The enrichment of $L$. casei procedure used in this work was based on a previously reported method with several modifications (Song et al., 2019b). Finally, $3 \mu \mathrm{L}$ of the cell suspension was pipetted out on parafilm and photographed under UV light using Bio-Rad GelDoc ${ }^{\mathrm{XR}}$ imaging system (Hercules, CA). For specificity assay, $10^{6}$ cells of each strain were used, and the operation was the same as described above. The nonviable cells of $L$. case $i$ were obtained by heating the cell suspension at $85^{\circ} \mathrm{C}$ for $10 \mathrm{~min}$, followed by subjecting to the detection operation.

\section{Detection of Viable L. casei Cells in Commercial Dairy Drinks}

Detection was carried out using a procedure based on the previous study with modifications (Song et al., 2019b). Six commercially available dairy drink brands, officially labeled with the content of viable $L$. casei, were analyzed using the established aptamer-based strategy. The drink sample of $10 \mathrm{~mL}$ was centrifuged at $16,000 \times g$ at $4^{\circ} \mathrm{C}$ for $10 \mathrm{~min}$ to isolate the cells from the beverage matrix. After discarding the supernatant, the precipitated cells were washed twice with PBS, and resuspended with $100 \mu \mathrm{L}$ of $1 \times \mathrm{BB}$ containing E-Apt of $0.05 \mu \mathrm{M}$. The resulting mixture was incubated at $37^{\circ} \mathrm{C}$ for $30 \mathrm{~min}$, followed by the addition of SMB of 10 $\mu \mathrm{L}$, and kept at room temperature for another $5 \mathrm{~min}$ with intermittent shaking. The SMB loading bacteria via E-Apt were then collected by magnet separation. The rest of the operation was the same as described in the above experimental section. Three samples from each brand were randomly picked, and the each sample was tested in triplicate.

\section{RESULTS}

\section{Principle of the Novel Cell-SELEX Strategy}

The detailed process of the established method was illustrated in Figure 1. First, incubation of ssDNA library with $L$. casei can guarantee the binding of the potential aptamers to the cells. The PC-GO was then introduced to adsorb nonspecific ssDNA and potential aptamers with low affinity. The ssDNA-loading $\mathrm{PC}-\mathrm{GO}$ was then removed and the CAC were dissociated from the cell surface by thermal denaturation. All the oligonucleotides used in this assay are listed in Supplemental Table S2 (https://doi.org/10.3168/jds .2019-16693). The CAC were amplified by CRM-RCA, and the single-stranded products were directly used as the aptamer pool for the next round of selection. The stepwise products from CRM-RCA are shown in Supplemental Figure S2 (https://doi.org/10.3168/jds .2019-16693). In total, 6 rounds of selection interposed by 2 rounds of counter selection were performed. The final aptamer pool was cloned and sequenced to obtain the definite sequence information.

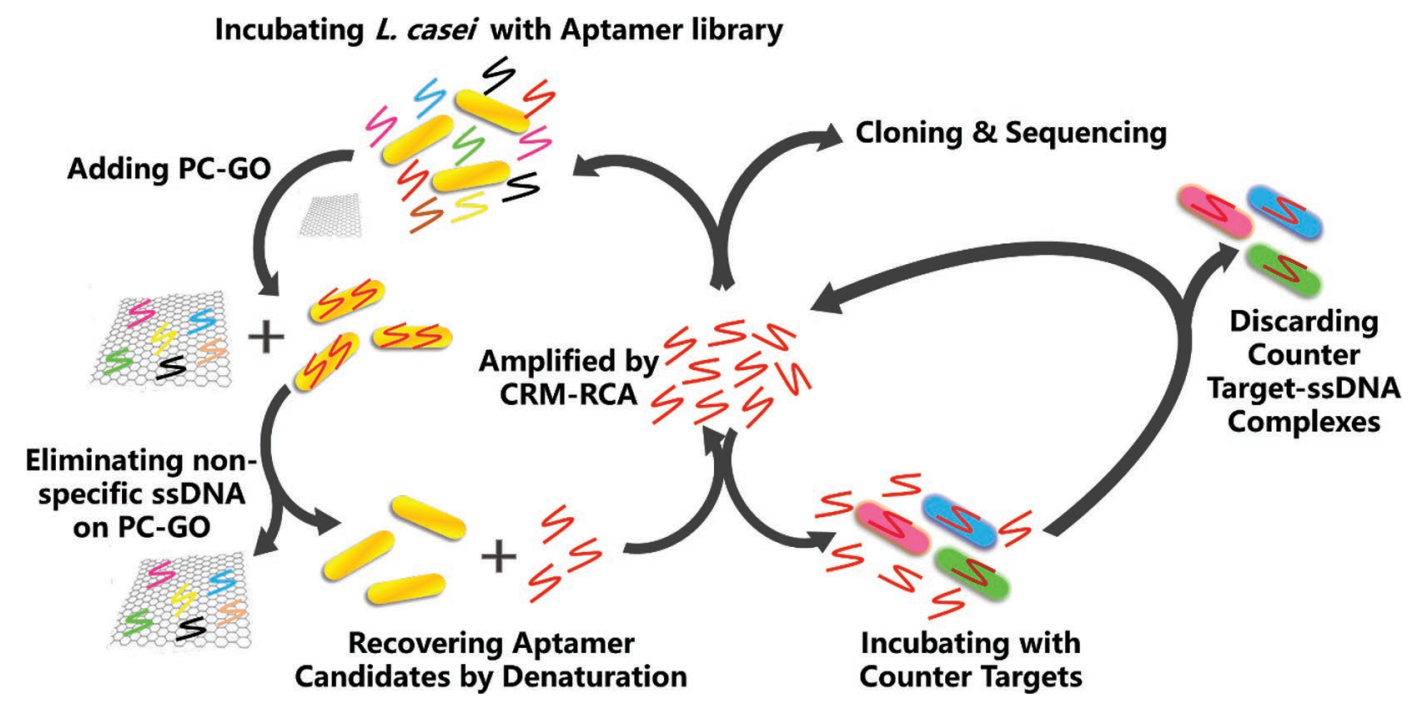

Figure 1. Schematic illustration of the aptamer screening procedure using a systematic evolution of ligands by exponential enrichment (SELEX) protocol using the whole bacterium cell as the target (cell-SELEX) powered by polyethyleneglycol and chitosan functionalized graphene oxide (PC-GO) and complementary ring-mediated rolling circle amplification (CRM-RCA). ssDNA = single-stranded DNA. L. = Lactobacillus. 
Table 1. Sequences of Lactobacillus casei-specific aptamers (Apt)

\begin{tabular}{llc}
\hline Name & Sequence $\left(5^{\prime}-3^{\prime}\right)$ & $\begin{array}{c}\text { Length }^{1} \\
(\mathrm{nt})\end{array}$ \\
\hline Apt-1 & ATCGTATACCTAGAAGATATGACCGGCAGTTGATGAGATAGAGGCGCTGGTGGGTAGATA & \\
Apt-2 & GGCTCACCTACAGGCTGCGGAGTCATAGCATCGTGACAGAGTCGAGTGTCGACTATACGT & 60 \\
Apt-3 & TCGAAGTGAGCGCGCGGTGTGGTGACTGTGTTGCAGATGGATGCATGGAGGTGATGATGA & 60 \\
\hline
\end{tabular}

${ }^{1} \mathrm{nt}=$ nucleotides.

\section{Specificities of the Selected Aptamers to L. casei}

After cloning and sequencing, 3 L. casei-specific aptamers were obtained and these sequences are summarized in Table 1. Flow cytometric analyses of incubation mixtures containing $5^{\prime}$ FITC-labeled aptamers and target cells were carried out to assess the specificity to L. casei. Lactobacillus acidophilus, S. thermophilus, L. bulgaricus, B. lactis, and L. plantarum, which are commonly used in dairy products, were applied here as references. As shown in Figure 2A, for all 3 aptamers, counts of $L$. casei in positive region were significantly higher than that of the other bacteria, indicating the selected aptamers are preferentially binding to $L$. casei. The relative positive rates of Apt-1, Apt-2, and Apt-3 for $L$. casei were $82.7,84.2$, and $88.5 \%$ respectively, whereas the values were less than $20 \%$ for the reference bacteria. It is worth noting that $B$. lactis and $L$. acidophilus were not involved in the counter selection, while the selected aptamers still showed lower affinities to them (Figure 2B), indicating that the aptamers are selectively binding to the unique protein or LPS-formed
A

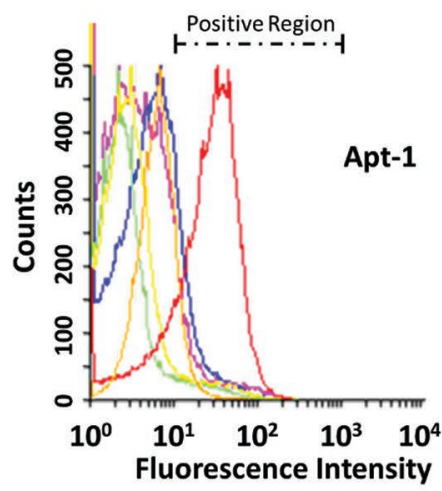

B

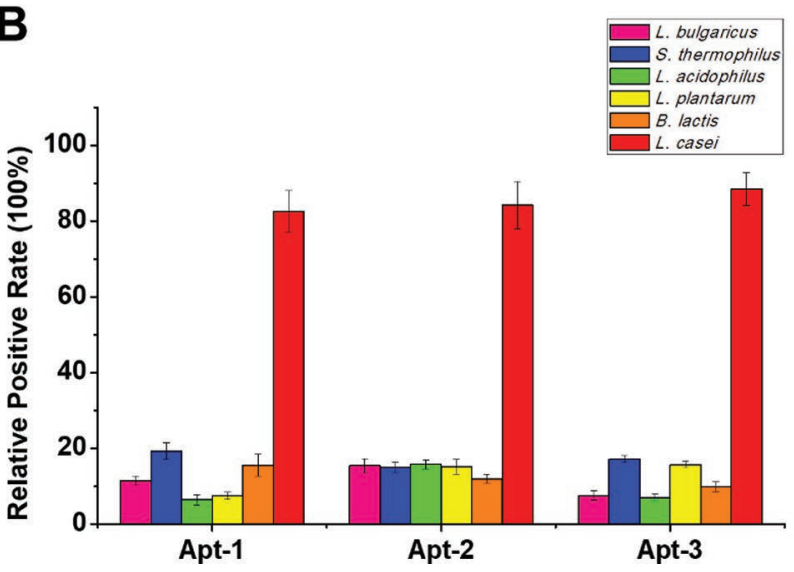

Apt-2
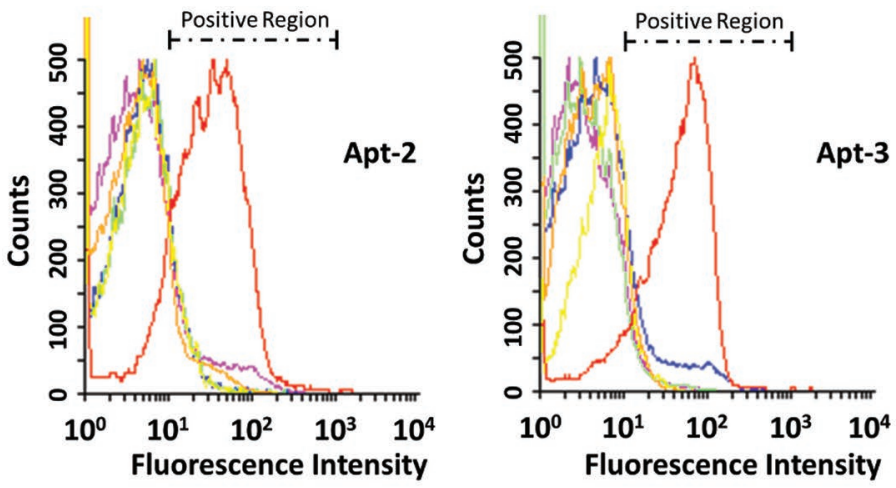

Figure 2. Affinity analysis of the selected aptamers (Apt) toward different bacteria strains. (A) Flow cytometry analysis of binding preference of the 3 Apt toward 6 types of bacterial cells. (B) Histogram summarizing the binding affinities in (A) in terms of relative positive rate designating fluorescence intensity in the range of 10 to $10^{3}$. The $5^{\prime}$ fluorescein isothiocyanate (FITC)-labeled Apt were used in the assay, whereas cells incubated with natural Apt served as the control to define background signal. Conditions: the 5' FITC-labeled Apt of $100 \mathrm{n} M$, incubated with $10^{6}$ cells at $37^{\circ} \mathrm{C}$ for $30 \mathrm{~min}$, followed by flow cytometry analysis. The error bars represent the SD of 3 parallel assays. 
tertiary structures on the $L$. casei cell surface (Shi et al., 2011). This guarantees that the aptamer-based strategy is of adequate specificity to identify $L$. casei among other probiotic or nonprobiotic strains in fermented milk products. Therefore, it can be concluded that the selected aptamers are highly specific to $L$. casei, which paves the way for the development of a robust detection strategy.

\section{$K_{d}$ and Secondary Structure}

of the L. casei-Specific Aptamers

The $K_{d}$ values were determined by incubating fluorescently labeled aptamers varying in concentration with a constant number of $L$. casei cells. The typical flow cytometry results ( $K_{d}$ for Apt-1) are shown in Figure $3 \mathrm{~A}$. The counts in the positive region significantly increased with the amount of Apt-1 at a lower concentration, yet the trend ceased when Apt-1 exceeded 100 $\mathrm{n} M$ (Figure 3B). The other 2 aptamers were observed with a saturation binding behavior as well, indicating that the interaction between the aptamer and the bacterium cell complied with ligand-competing mechanism (Amano et al., 2016). Based on nonlinear regression curve fitting, $K_{d}$ values were estimated as $21.1 \pm 2.7$, $19.3 \pm 3.7$, and $15.6 \pm 4.1 \mathrm{n} M$ for Apt-1, Apt-2, and Apt-3, respectively, which were comparable to aptamers against bacteria cells reported previously (Davydova et al., 2016). Figure 3C summarizes the estimated $K_{d}$ values and the secondary structures (predicted by
A

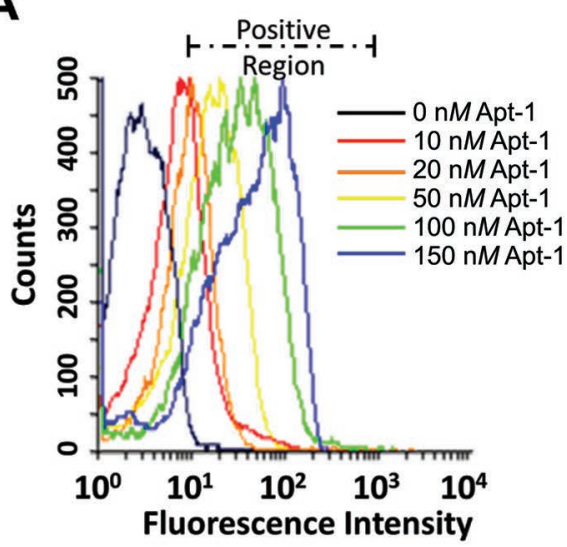

B

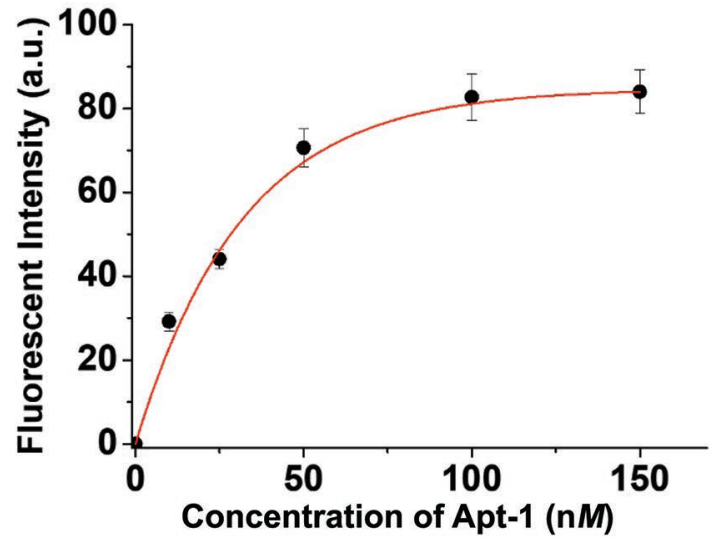

C

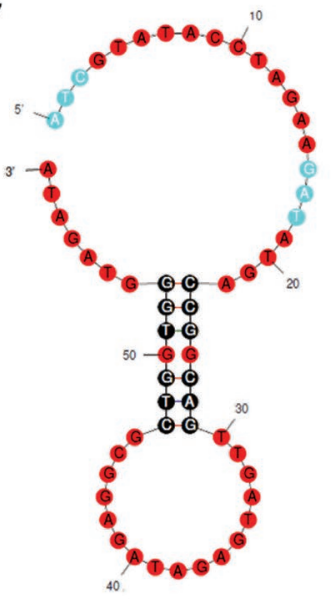

$\Delta G=-3.53 \mathrm{~kJ} / \mathrm{mol}$

$\mathrm{K}_{\mathrm{d}}=21.1 \pm 2.7 \mathrm{n} M$ Apt-1

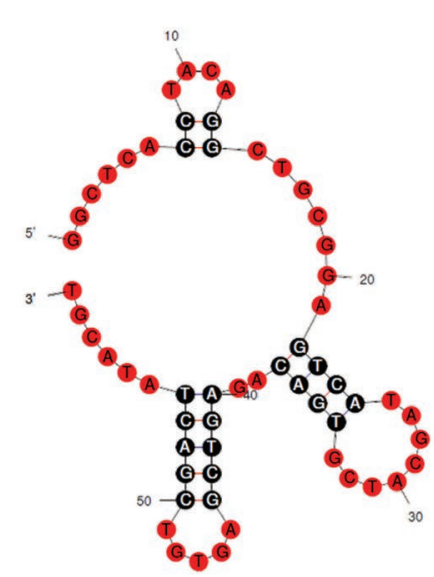

$\Delta G=-8.2 \mathrm{~kJ} / \mathrm{mol}$ $\mathrm{K}_{\mathrm{d}}=19.3 \pm 3.7 \mathrm{nM}$ Apt-2

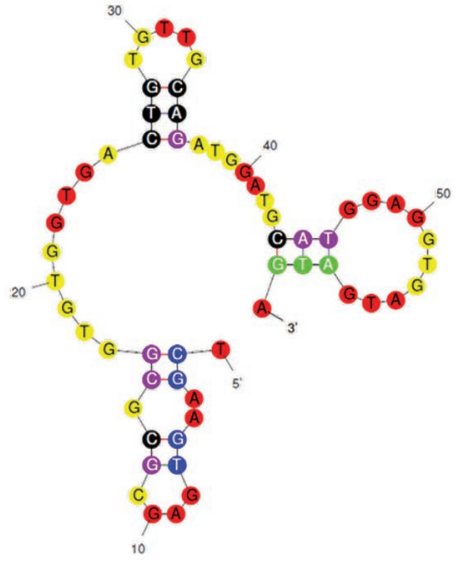

$\Delta G=-8.33 \mathrm{~kJ} / \mathrm{mol}$ $K_{d}=15.6 \pm 4.1 \mathrm{nM}$ Apt-3

Figure 3. The dissociating constant $\left(K_{d}\right)$ values and the predicted secondary structures of the selected aptamers (Apt). (A) Flow cytometry results of Lactobacillus casei cells binding with the Apt varying in concentration. (B) Saturation curve of Apt-1 binding to the target cells. A nonlinear regression curve was applied to fit the experimental data demonstrated in (A). (C) The predicted secondary structures and $K_{d}$ values of the Apt. Conditions for $K_{d}$ assay: cells of $10^{6}$, fluorescein isothiocyanate (FITC)-labeled Apt of concentration from 0 to $150 \mathrm{n} M$, incubated at $37^{\circ} \mathrm{C}$ for $30 \mathrm{~min} . \Delta G=$ free energy of formation. The error bars represent the SD of 3 parallel assays. 
mfold software) of the selected aptamers for $L$. casei. These structures were highly self-complementing, especially with some stem-loop motifs usually found in riboswitches (Weinberg et al., 2017). The $\Delta G$ values were negative under the ambient conditions, suggesting that those structures can spontaneously form, which serves as the basis for binding to $L$. casei cells.

\section{Analysis of L. casei Cells in Viable and Nonviable Conditions Using the Aptamer-Based Strategy}

Two L. casei-specific aptamers, one for enriching the bacteria (E-Apt) and the other for fluorescent detection (D-Apt), were applied to build the strategy. Sequence of the oligomers used in the detection are shown in Table 2. A poly(A) tail was attached to the $3^{\prime}$ terminus of E-Apt to provide certain rigidity to the sequence, a property that reduces cross-paring of the aptamer sequences when bound to the magnetic beads. The other 5 bacteria, usually found in probiotic products, were used here as the references to verify the specificity for L. casei analysis. As shown in Figure 4A, the sample containing $L$. casei rendered a dramatic fluorescent signal, whereas the other bacteria (except for L. plantarum) were observed with fluorescent intensities not significantly different from the nonbacterium control. The mean fluorescent intensities of 3 parallel samples are presented in the bar chart, where the fluorescent intensity of the target cell was at least 10-fold higher than that of the other reference bacteria, demonstrating again that the aptamer was selectively binding to the unique surface structure of $L$. casei. To confirm the detection results, a L. casei gene-specific PCR assay was conducted and the results were consistent with that presented by the current ones (Supplemental Figure S3, Supplemental Information, https://doi.org/ 10.3168/jds.2019-16693), indicating the aptamer-based strategy was of sufficient specificity to identify the $L$. casei strain. The high specificity could be ascribed to the fact that only $L$. casei cells can be simultaneously recognized by the 2 aptamers and resulted in fluorescent signals for detection. For L. plantarum, fluorescent intensity higher than that of other 4 reference bacteria was observed, a phenomenon that was probably caused by the partially shared membrane protein motif between L. casei and L. plantarum (Shao et al., 2015; $\mathrm{Wu}$ et al., 2016). However, this fluorescent intensity was particularly low in comparison to that rendered by L. casei cells, ensuring the detection specificity of the established strategy.

The sensitivity of the strategy was studied by using serial diluted $L$. casei cell suspensions $\left(10^{9}\right.$ to $10^{4}$ $\mathrm{cfu} / \mathrm{mL}$ ). As can be seen in Figure 4B, the fluorescent intensity declined with the decreasing concentration of the target cells, indicating that the signal was in direct proportional to the L. casei concentration. No fluorescence signal could be detected when the cell concentration dropped down to $10^{4} \mathrm{cfu} / \mathrm{mL}$; thus, the detection limit of the present strategy was determined as $10^{5} \mathrm{cfu} /$ $\mathrm{mL}$. A linear correlation curve in the range of detection $\left(10^{9}\right.$ to $\left.10^{5} \mathrm{cfu} / \mathrm{mL}\right)$ was also obtained as shown in Figure 4C. Although the limit of detection was higher than that of the aptamer-based strategies reported previously (Duan et al., 2015; Wu et al., 2015), the present method is still adequate for quantitative analysis of $L$. casei since virtually all $L$. casei-related dairy products are labeled with cell numbers greater than $10^{6} \mathrm{cfu} / \mathrm{mL}$. Besides, L. casei in viable and nonviable conditions can also be distinguished based on fluorescent intensity. As shown in Figure 4D, in the range of $10^{9}$ to $10^{6} \mathrm{cfu} / \mathrm{mL}$, the viable $L$. casei cells were detected with fluorescent intensity significantly higher than that of nonviable ones. One exception was $10^{5} \mathrm{cfu} / \mathrm{mL}$, where the difference was insignificant. This was probably caused by the reduced fluorescence quantum yield of FITC when used at lower concentrations (Resch-Genger et al., 2008). Lactobacillus casei cells in the nonviable state can still render detectable fluorescent signals, which could be ascribed to the renaturation of some membrane proteins after heat deactivation (Renthal, 2006). In fact, this barely has any adverse effects on viable cell enumeration, since 4-time differences were obtained in the range of $10^{9}$ to $10^{6} \mathrm{cfu} / \mathrm{mL}$. Hence, the established strategy was proved with desirable specificity and sensitivity to quantitatively identify viable $L$. casei cells, which paves the way for the application on commercial dairy drinks.

Table 2. Sequence of oligomers used in the detection ${ }^{1}$

\begin{tabular}{lll}
\hline Name & Sequence $\left(5^{\prime}-3^{\prime}\right)$ & $\begin{array}{c}\text { Length }^{2} \\
(\text { nt })\end{array}$ \\
\hline E-Apt & GGCTCACCTACAGGCTGCGGAGTCATAGCATCGTGACAGAGTCGAGTGTCGACTATACGTAAAAAAAAAA-b & 70 \\
D-Apt & FITC-TCGAAGTGAGCGCGCGGTGTGGTGACTGTGTTGCAGATGGATGCATGGAGGTGATGATGA & 60 \\
\hline $\begin{array}{l}{ }^{1} \text { E-Apt }=\text { enriching aptamer; D-Apt }=\text { detecting aptamer. b represents a biotin modification. FITC indicates a fluorescein isothiocyanate group } \\
\text { for fluorescent detection. }\end{array}$ & \\
${ }^{2} \mathrm{nt}=$ nucleotides.
\end{tabular}


A

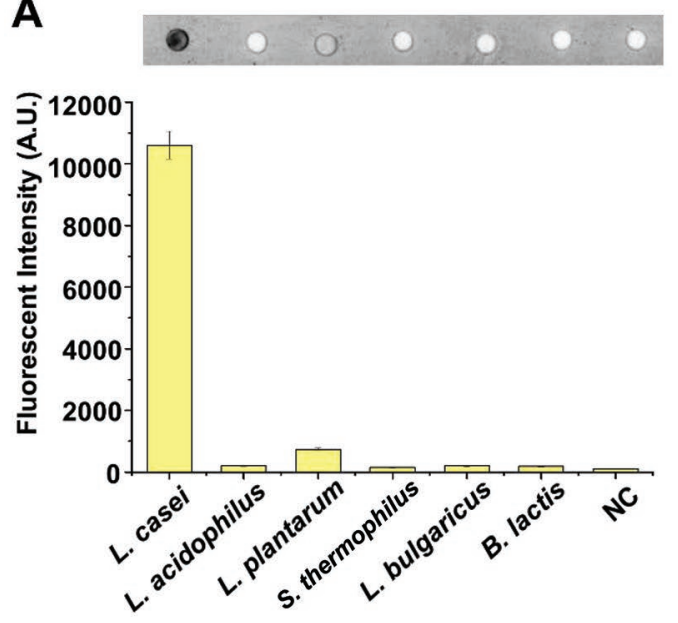

C

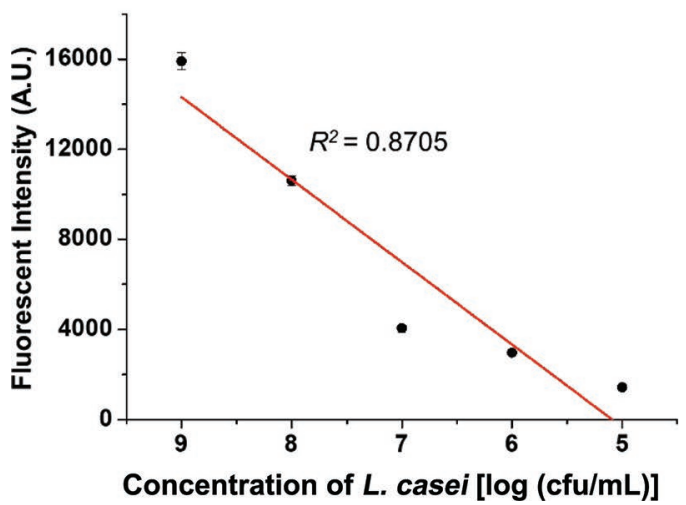

B
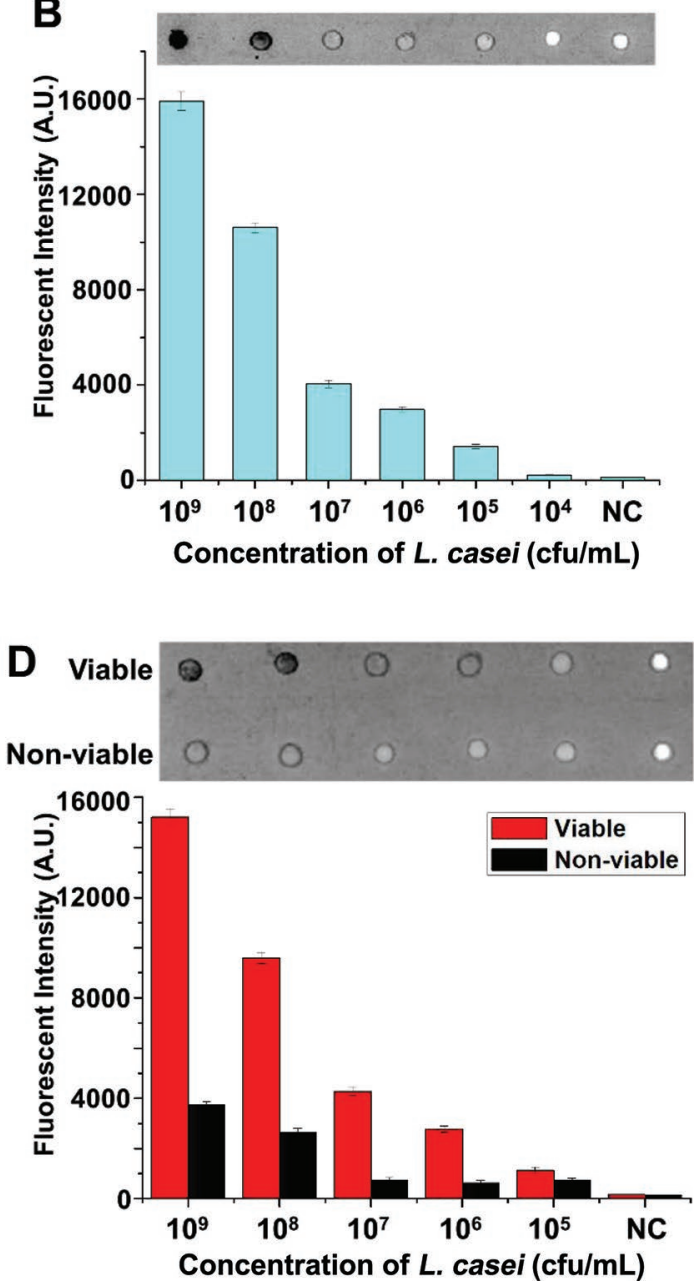

Figure 4. Analysis of Lactobacillus casei by using the aptamer-based strategy. (A) Specificity of the aptamer-based strategy toward different types of bacteria cells. Conditions: cells of $10^{6}, 50 \mathrm{nM}$ fluorescein isothiocyanate (FITC)-labeled aptamer, incubated at $37^{\circ} \mathrm{C}$ for 30 min. $\mathrm{NC}=$ nonbacterium control. (B) Sensitivity of the aptamer-based strategy for L. casei detection. (C) The linear correlation curve between bacterium concentration and detected fluorescent intensity. $\mathrm{R}^{2}$ represents the value of the coefficient of determination between $L$. casei concentration and fluorescent intensity. (D) Detection of viable and nonviable L. casei varying in concentration using the aptamer-based strategy. A.U. = arbitrary units. Error bars represent SD of 3 parallel assays.

\section{Practice Application on Viable L. casei Analysis in Dairy Drinks}

Lactobacillus casei is added to several commercially available dairy products, specially fermented drinks, to provide probiotic effects. Most of the manufacturers claimed a concentration of more than $10^{6} \mathrm{cfu} / \mathrm{mL}$ of $L$. casei cells in viable condition. In some premium products, the number is $10^{8} \mathrm{cfu} / \mathrm{mL}$. Here, the aptamerbased strategy was applied to identify and quantify $L$. casei in commercially available fermented milk beverages. Six brands of the beverages were selected, and 3 randomly chosen samples of each brand were subjected to the analysis. The quantity of $L$. casei cells in each sample was determined based on the linear correlation curve plotted by using $L$. casei with known concentrations against corresponding fluorescent intensities (Supplemental Figure S4, Supplemental Information, https://doi.org/10.3168/jds.2019-16693). The accurate number of $L$. casei cells in the dairy products can also be obtained according to the correlation curve represented in Supplemental Figure S4. The analysis results and the product information regarding days until expiration are listed in Table 3. Qualitatively, all of the samples were detected containing viable $L$. casei cells on account of the significantly high fluorescent signals (Supplemental Table S3, Supplemental Information, https://doi.org/10.3168/jds.2019-16693). The average fluorescent intensities of the 18 samples analyzed by the aptamer-based strategy are shown in Supplemental 
Table 3. Results of analyzing Lactobacillus casei in dairy drinks using the aptamer-based strategy ${ }^{1}$

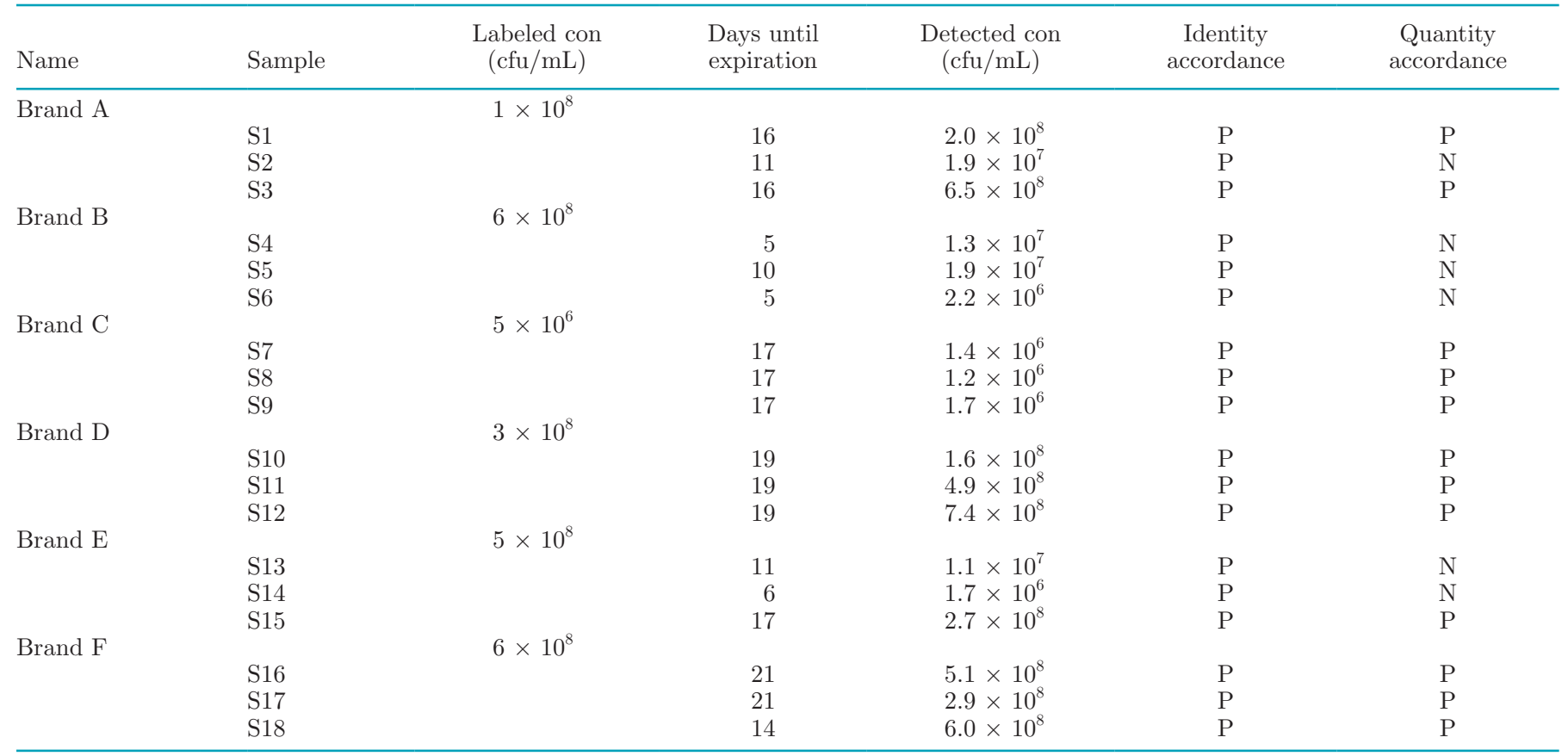

${ }^{1} \mathrm{P}=$ positive analysis result; $\mathrm{N}=$ negative analysis result; con = concentration. The condition in which the exponent part of the detection value is the same as that of the labeled concentration is considered positive in terms of quantity accordance.

Figure S5 (https://doi.org/10.3168/jds.2019-16693). Hence, identity accordance of the 18 samples from the 6 brands was determined as positive. However, quantity accordance of the 18 samples largely varied. For brand A, the labeled concentration of $L$. case $i$ was $1 \times 10^{8}$ $\mathrm{cfu} / \mathrm{mL}$, which was negated by the fact that one of its samples (sample 2) was determined to have $1 \times 10^{7} \mathrm{cfu} /$ mL. All 3 samples (samples 4, 5, and 6) from brand B were determined as at least one magnitude lower than the labeled concentrations, suggesting a possibility of quantity discordance in this batch. Samples 13 and 14 from brand E were also detected with $L$. casei amount less than claimed. The discordance in L casei amount might be ascribed to expiration or improper storage conditions because some of the samples (samples 4, 6, and 14) were indeed on the verge of their shelf lives. To confirm the samples with discordance, a classic platecounting method was used (Supplemental Figure S6, Supplemental Information, https://doi.org/10.3168/ jds.2019-16693). The results were in line with that provided by the established strategy, suggesting that the method is promising to be used as a preliminary screening approach for food surveillance.

\section{DISCUSSION}

Rapid identification and enumeration of a valuable probiotics in food products are of great significance for food quality assurance and market monitoring. Plate counting on selective medium and specific gene detection by PCR are classic methods to fulfill these tasks (Temmerman et al., 2004). However, these protocols are complicated and time consuming, and some of them are not able to distinguish viable and nonviable cells. Here, a novel aptamer-based strategy was developed to qualitatively and quantitatively analyze $L$. casei in dairy drinks. Aptamer is a ssDNA or RNA oligomer that can specifically bind to targets with affinity comparable to that of antibody. Three $L$. casei-specific aptamers were obtained in this study by using PC-GO and CRM-RCA powered cell-SELEX. The selected aptamers are preferentially binding to $L$. casei, and showing lower affinities toward other LAB strains (Figure 2). As for the similar strains mainly including Lactobacillus paracasei and Lactobacillus rhamnosus, an in-depth study is needed to investigate whether the $L$. case $i$-specific aptamers would react to them. Based on the aptamers, the presence of $L$. casei was selectively detected by the established strategy (Figure 4A), which avoided the long-time bacteria culture and subsequent identification procedure in the plate counting method (Singh et al., 2009). The aptamers bind to the tertiary unique structures on the surface of $L$. casei, generating visualized fluorescent signals for rapid detection. The whole process can be accomplished within $1.5 \mathrm{~h}$, free of DNA extraction or purification that is necessary for 
PCR-based detection strategies (Furet et al., 2004; Herbel et al., 2013). Therefore, the established strategy has proven efficiency and reliability, enabling it to be used as powerful tool for food inspection and supervision. Since only 2 aptamer sequences and magnetic beads are used to qualitatively and quantitatively analyze the amount of $L$. casei, the cost per sample of the established strategy was less than $\$ 1$ based on rough calculation. The expense is much lower than that of PCR, fluorescence in situ hybridization (FISH), and quantitative PCR, which require costly DNA polymerases, nucleic acid probes, and quantitative PCR mix (Haarman and Knol, 2006; Binetti et al., 2008; Machado et al., 2013). Therefore, rapid and high-throughput analysis can be expected using techniques based on the current strategy. The limit of detection of the present protocol is determined as $10^{5}$ $\mathrm{cfu} / \mathrm{mL}$, which is adequate for quantitative analyses of L. casei in commercially available drinks. The detection limit can be easily enhanced by modifying the signal report aptamer (D-Apt) with quantum dots, for their high fluorescence quantum yield and low irradiation decay (Michalet, 2005). Furthermore, simultaneously detecting different bacteria can be realized by using fluorophores or quantum dots varying in excitation or emission wavelength (Duan et al., 2013).

When we first applied the developed strategy for $L$. casei in dairy products, ingredients from the beverage matrix such as lactoprotein and glucan interfered with the binding between the aptamer and the target cell, resulting in false-negative results. Hence, centrifugation is applied to eliminate the beverage matrix. As inspired by the paper-made centrifuge reported recently, the entire analysis operation is promising to be integrated into a small and portable device, which will further enable the strategy for on-the-spot inspection (Bhamla et al., 2017).

The aptamer-based strategy is capable of discriminating between viable and nonviable $L$. casei cells, based on the fact that drastic change of the surface structure ensues once bacteria are dead. We first expected that nonviable $L$. casei would fail to generate detectable signals. However, it is somewhat surprising that the thermal sterilized $L$. casei cells are still observed with fluorescent signals (Figure 4D). A possible reason for this is the renaturation of some transmembrane proteins, resulting in original protein motifs available for D-Apt binding (Kort et al., 2005; Renthal, 2006). In practice, bacterial death is often associated with improper storage conditions (fluctuant temperature, intensive irradiation, and so on), where the cells undergo a serial process of cell wall collapsing, surface protein decomposing, and nuclear DNA degrading (Rice and Bayles, 2008). Therefore, these nonviable cells could be clearly discriminated from the viable ones by using the aptamer-based strategy. Related studies to promote the practical applications of the established strategy are now in progress.

\section{CONCLUSIONS}

In summary, highly specific aptamers preferentially targeting $L$. casei cells were obtained by a novel cellSELEX protocol utilizing functionalized $\mathrm{GO}$ and RCA-based isothermal amplification. The 3 aptamers are selectively binding to $L$. casei rather than other LAB strains, based on which a robust detection strategy was built with the ability to selectively detect $L$. casei in a linear range of $10^{5}$ to $10^{9} \mathrm{cfu} / \mathrm{mL}$. In addition, viable and nonviable cells can be discriminated by the established strategy due to the ability of the aptamers to recognize the change of the tertiary surface structures caused by bacterial death. Practicability of the aptamer-based strategy was demonstrated by the randomly chosen samples from 6 commercial brands of dairy products. To the best of our knowledge, this is the first report of $L$. case $i$-specific aptamers and the related strategy for qualitative and quantitative analysis of viable cells in dairy drinks. The method could be used to qualitatively and quantitatively analyze viable L. casei in dairy products for quality management and food regulation ends. Further studies analyzing $L$. casei in yogurt and cheese using a high-speed homogenizer and ultrasonic generator are underway.

\section{ACKNOWLEDGMENTS}

This study was supported by grants from the National Natural Science Foundation for Young Scholar of China (81601877), National Natural Science Foundation of China (31671823), Key R\&D Program of Shaanxi Province (2018NY-095), and Fundamental Research Funds for the Central Universities (GK201603101, China).

\section{REFERENCES}

Amano, R., K. Takada, Y. Tanaka, Y. Nakamura, G. Kawai, T. Kozu, and T. Sakamoto. 2016. Kinetic and thermodynamic analyses of interaction between a high-affinity RNA aptamer and its target protein. Biochemistry 55:6221-6229.

Bagheripoor-Fallah, N., A. Mortazavian, H. Hosseini, S. Khoshgozaranabras, and A. H. Rad. 2015. Comparison of molecular techniques with other methods for identification and enumeration of probiotics in fermented milk products. Crit. Rev. Food Sci. Nutr. 55:396-413.

Bhamla, M. S., B. Benson, C. Chai, G. Katsikis, A. Johri, and M. Prakash. 2017. Hand-powered ultralow-cost paper centrifuge. Nat. Biomed. Eng. 1:0009.

Binetti, A. G., M. L. Capra, M. A. Álvarez, and J. A. Reinheimer. 2008. PCR method for detection and identification of Lactobacillus casei/paracasei bacteriophages in dairy products. Int. J. Food Microbiol. 124:147-153. 
Boylston, T. D., C. G. Vinderola, H. B. Ghoddusi, and J. A. Reinheimer. 2004. Incorporation of bifidobacteria into cheeses: Challenges and rewards. Int. Dairy J. 19:315-387.

Davis, C. 2014. Enumeration of probiotic strains: Review of culturedependent and alternative techniques to quantify viable bacteria. J. Microbiol. Methods 103:9-17.

Davydova, A., M. Vorobjeva, D. Pyshnyi, S. Altman, V. Vlassov, and A. Venyaminova. 2016. Aptamers against pathogenic microorganisms. Crit. Rev. Microbiol. 42:847-865.

Duan, N., S. Wu, S. Dai, T. Miao, J. Chen, and Z. Wang. 2015. Simultaneous detection of pathogenic bacteria using an aptamer based biosensor and dual fluorescence resonance energy transfer from quantum dots to carbon nanoparticles. Mikrochim. Acta 182:917923.

Duan, N., S. Wu, Y. Yu, X. Ma, Y. Xia, X. Chen, Y. Huang, and Z. Wang. 2013. A dual-color flow cytometry protocol for the simultaneous detection of Vibrio parahaemolyticus and Salmonella typhimurium using aptamer conjugated quantum dots as labels. Anal. Chim. Acta 804:151-158.

Duan, Y., Z. Gao, L. Wang, H. Wang, H. Zhang, and H. Li. 2016. Selection and identification of chloramphenicol-specific DNA aptamers by mag-SELEX. Appl. Biochem. Biotechnol. 180:1644-1656.

FAO/WHO. 2002. Guidelines for the evaluation of probiotics in food report of a joint FAO/WHO Working Group. London, Ontario, Canada: Food and Agriculture Organization of the United Nations and World Health Organization.

Furet, J. P., P. Quénée, and P. Tailliez. 2004. Molecular quantification of lactic acid bacteria in fermented milk products using real-time quantitative PCR. Int. J. Food Microbiol. 97:197-207.

Haarman, M., and J. Knol. 2006. Quantitative real-time PCR analysis of fecal Lactobacillus species in infants receiving a prebiotic infant formula. Appl. Environ. Microbiol. 72:2359-2365.

Herbel, S. R., B. Lauzat, M. V. Nickisch-Rosenegk, M. Kuhn, and S. Guenther. 2013. Species-specific quantification of probiotic lactobacilli in yoghurt by quantitative real-time PCR. J. Appl. Microbiol. 115:1402-1410.

Jankovic, I., W. Sybesma, P. Phothirath, E. Ananta, and A. Mercenier. 2010. Application of probiotics in food products-challenges and new approaches. Curr. Opin. Biotechnol. 21:175-181.

Kort, R., A. C. O'Brien, I. H. M. Van Stokkum, S. J. C. M. Oomes, W. Crielaard, K. J. Hellingwerf, and S. Brul. 2005. Assessment of heat resistance of bacterial spores from food product isolates by fluorescence monitoring of dipicolinic acid release. Appl. Environ. Microbiol. 71:3556-3564.

Kourkoutas, Y., V. Xolias, M. Kallis, E. Bezirtzoglou, and M. Kanellaki. 2005. Lactobacillus casei immobilization on fruit pieces for probiotic additive, fermented milk and lactic acid production. Process Biochem. 40:411-416.

Lee, Y. J., S. R. Han, J. S. Maeng, Y. J. Cho, and S. W. Lee. 2012. In vitro selection of Escherichia coli O157:H7-specific RNA aptamer. Biochem. Biophys. Res. Commun. 417:414-420.

Machado, A., C. Almeida, A. Carvalho, F. Boyen, F. Haesebrouck, L Rodrigues, N. Cerca, and N. F. Azevedo. 2013. Fluorescence in situ hybridization method using a peptide nucleic acid probe for identification of Lactobacillus spp. in milk samples. Int. J. Food Microbiol. 162:64-70.

Michalet, X. 2005. Quantum dots for live cells, in vivo imaging, and diagnostics. Science 307:538-544.
Reid, G., and A. Bocking. 2003. The potential for probiotics to prevent bacterial vaginosis and preterm labor. Am. J. Obstet. Gynecol. 189:1202-1208.

Renthal, R. 2006. An unfolding story of helical transmembrane proteins. Biochemistry 45:14559-14566.

Resch-Genger, U., M. Grabolle, S. Cavalierejaricot, R. Nitschke, and T. Nann. 2008. Quantum dots versus organic dyes as fluorescent labels. Nat. Methods 5:763-775.

Rice, K. C., and K. W. Bayles. 2008. Molecular control of bacterial death and lysis. Microbiol. Mol. Biol. Rev. 72:85-109.

Sefah, K., D. Shangguan, X. Xiong, M. B. O'Donoghue, and W. Tan. 2010. Development of DNA aptamers using Cell-SELEX. Nat. Protoc. 5:1169-1185.

Shao, Y., W. Zhang, H. Guo, L. Pan, H. Zhang, and T. Sun. 2015. Comparative studies on antibiotic resistance in Lactobacillus casei and Lactobacillus plantarum. Food Control 50:250-258.

Shi, H., X. He, K. Wang, X. Wu, X. Ye, Q. Guo, W. Tan, Z. Qing, X. Yang, and B. Zhou. 2011. Activatable aptamer probe for contrast-enhanced in vivo cancer imaging based on cell membrane protein-triggered conformation alteration. Proc. Natl. Acad. Sci. USA 108:3900-3905.

Singh, S., P. Goswami, R. Singh, and K. J. Heller. 2009. Application of molecular identification tools for Lactobacillus, with a focus on discrimination between closely related species: A review. Lebensm. Wiss. Technol. 42:448-457.

Sohrabvandi, S., S. H. Razavi, S. M. Mousavi, and A. M. Mortazavian. 2010. Viability of probiotic bacteria in low alcohol- and non-alcoholic beer during refrigerated storage. Philipp. Agric. Sci. 93:24-28.

Song, S., X. Wang, K. Xu, Q. Li, L. Ning, and X. Yang. 2019a. Selection of highly specific aptamers to Vibrio parahaemolyticus using cell-SELEX powered by functionalized graphene oxide and rolling circle amplification. Anal. Chim. Acta 1052:153-162.

Song, S., X. Wang, K. Xu, Q. Li, G. Xia, and X. Yang. 2019b. Visualized detection of Vibrio parahaemolyticus in food samples using dual-functional aptamers and cut-assisted rolling circle amplification. J. Agric. Food Chem. 67:1244-1253.

Temmerman, R., G. Huys, and J. Swings. 2004. Identification of lactic acid bacteria: Culture-dependent and culture-independent methods. Trends Food Sci. Technol. 15:348-359.

Weinberg, Z., J. W. Nelson, C. E. Lünse, M. E. Sherlock, and R. R. Breaker. 2017. Bioinformatic analysis of riboswitch structures uncovers variant classes with altered ligand specificity. Proc. Natl. Acad. Sci. USA 114:E2077-E2085.

Wu, S., Y. Wang, N. Duan, H. Ma, and Z. Wang. 2015. Colorimetric aptasensor based on enzyme for the detection of Vibrio parahaemolyticus. J. Agric. Food Chem. 63:7849-7854.

Wu, Z., P. Wang, J. He, D. Pan, X. Zeng, and J. Cao. 2016. Proteome analysis of Lactobacillus plantarum strain under cheese-like conditions. J. Proteomics 146:165-171.

Yadav, H., S. Jain, and P. R. Sinha. 2007. Antidiabetic effect of probiotic dahi containing Lactobacillus acidophilus and Lactobacillus casei in high fructose fed rats. Nutrition 23:62-68.

\section{ORCIDS}

Xingyu Wang @ https://orcid.org/0000-0003-4000-464X 